\title{
Immune potentiator for increased safety and improved protection of vaccines by NF-kB modulation
}

Authors: Brittany A. Moser ${ }^{1}$, Rachel C. Steinhardt ${ }^{1}$, Yoseline Escalante-Buendia ${ }^{1}$, David A. Boltz $^{2}$, Kaylynn M. Barker ${ }^{2}$, Stan Yoo ${ }^{3}$, Bethany G. McGonnigal ${ }^{3}$, Aaron P. Esser-Kahn*1

\begin{abstract}
Affiliations:
${ }^{1}$ Institute for Molecular Engineering, University of Chicago, 5640 South Ellis Avenue, Chicago, Illinois 60637, USA.

${ }^{2}$ Division of Microbiology and Molecular Biology, IIT Research Institute, Illinois Institute of Technology, 10W. 35th Street, Chicago, IL 60616, USA

${ }^{3}$ Department of Chemistry, Chemical Engineering \& Materials Science, Biomedical Engineering, University of California, Irvine, California 92697, USA

*Correspondence to: aesserkahn@uchicago.edu
\end{abstract}

Abstract: Many modern vaccines include adjuvants that activate the immune system and provide an enhanced humoral or cellular response. Current approved adjuvants are unable to provide desired responses against some pathogens (e.g. HIV or dengue). Many new adjuvants have been developed and demonstrate promising results, but side effects from the inflammatory response induced by these adjuvants have resulted in limited FDA approvals. No adjuvants yet possess the capability to independently modulate inflammation and protection. Here we demonstrate a method to limit inflammation and side effects associated with vaccination while

20 retaining the protective responses using a variety of promising adjuvants. To accomplish this, we combined a selective NF-kB inhibitor with the immune adjuvant. The resulting vaccines reduce systemic inflammation and boost antibody responses. In an influenza challenge model, we demonstrate that this approach enhances protection. This method is generalizable across a broad range of adjuvants and antigens. We anticipate these studies will lead to a novel approach to

25 vaccine formulation design that may prove general across a wide range of adjuvants, enabling their greater use in the public realm.

Main Text: Vaccines are considered one of the most effective global health interventions against infectious diseases. Despite their success, current and future vaccines face contradictory challenges of increasingly stringent safety margins and more effective and diverse protective responses. A major challenge in developing new vaccine approaches is striking a balance between effective immune activation, leading to protective responses, and limiting the excess inflammation and side effects. To boost the immune response, toll-like receptor (TLR) agonists have been explored as vaccine adjuvants because they activate the innate immune system, promoting the expression of inflammatory cytokines and cell surface receptors important for T cell interactions (1-6). Effective TLR agonists stimulate the desired cellular or humoral adaptive responses; however, the 
inflammation induced by many of these compounds has made it challenging to transition them into new clinical vaccines (7). For example, CpG DNA, a TLR 9 agonist, has wide-ranging promise as a vaccine adjuvant and provides protection for diseases currently without a vaccine, such as HIV (8). CpG DNA also enables vaccines to be produced with less antigen (9), induces protective responses faster (10), and produces effective anti-tumor activity $(11,12)$. However, the excessive inflammatory response induced by this adjuvant has resulted in many clinical trial failures and is cited as limiting its therapeutic promise $(13,14)$. CpGs are only a fraction of the hundreds of TLR agonists (15). However due to the unsafe side effects, only a handful of TLR agonists are approved for limited use in humans (16). Studies indicate that side effects are mediated through systemic distribution of TNF-a and IL-6 (17,18). Here we demonstrate a method to decouple part of the inflammatory response from the antigen presenting actions of several adjuvants using an immune potentiator. Using a broad range of TLR agonists, we demonstrate both in vitro and in vivo that using an immune potentiator decreases proinflammatory cytokines while maintaining adaptive immune function. In vivo, we find that co-administering the immune potentiator with the 20172018 flu vaccine (Fluzone) decreases side effects associated with vaccination and increases protection. Co-administration of the immune potentiator with CpG-ODN1826 (CpG) and dengue capsid protein leads to elimination of systemic proinflammatory cytokines post-vaccination and yields increased, neutralizing antibodies. Additionally, administering the immune potentiator with CpG and gp120, a HIV viral coat protein, increased serum IgG and vaginal IgA antibodies and shifted IgG antibody epitope recognition. Lastly, we observed immune potentiation for several TLR agonists - implying a general approach. Immune-potentiation may find use in reducing the systemic side effects associated with inflammation for many adjuvanted vaccines (19) - creating the potential for many PRR agonists to be used safely, increasing the diversity of adaptive immune profiles and widening the scope of disease prevention and treatment.

\section{Selection of Immune Potentiator}

In seeking a method of immune potentiation, we explored the extensive research on the TLR activation pathway. This powerful mechanistic framework let us hypothesize about how TLR activation directs inflammatory cytokines and antigen presentation. As TLR pathways converge with NF-kB activation, and inflammatory and adaptive responses diverge upon which NF-kB subunit is activated, we hypothesized that we could decouple these processes via selective inhibition - leading to reduced side effects but maintaining the adaptive response. Upon TLR 
activation, the transcription factor NF-kB primes the transcription of pro-inflammatory cytokines such as IL-6 and TNF-a, and cell surface receptors such as MHC-II, CD40, CD80 and CD86 (2022). The NF-kB family is a family of transcription factors, consisting of two subunits: a DNA binding domain and a transcriptional activator $(23,24)$. Each NF-kB dimer controls expression of a different set of genes for distinct cellular processes - broadly, some dimers control inflammatory expression while others control antigen presentation (23-25). Selectively modulating a pathway, we conjectured, might lead to increased antigen presentation, while decreasing inflammation. NF$\mathrm{kB}$ inhibitors have been widely explored for reducing cytokine expression in cancer (26-29), autoimmune disorders $(30,31)$, and sepsis (32-34), yet they have not been explored as vaccine potentiators. This lack of experimentation may be because it is broadly understood that NF- $\kappa \mathrm{B}$ activation is necessary in mounting an adequate adaptive immune response $(29,35)$. However, only certain subunits direct antigen presentation (36). As a proof-of-concept immune potentiator we chose SN50, a cell permeable peptide that consists of the nuclear localization sequence (NLS) of the NF-kB subunit, p50 which blocks the import of p50 containing dimers into the nucleus (37). First, we sought to determine if SN50 enables inhibition of NF-kB of innate immune cells. We validated that SN50 reduced total NF-kB activity in human (THP-1 monocytes) and mouse (RAW macrophages) cells in a dose dependent manner. (Fig.S1a-d). 

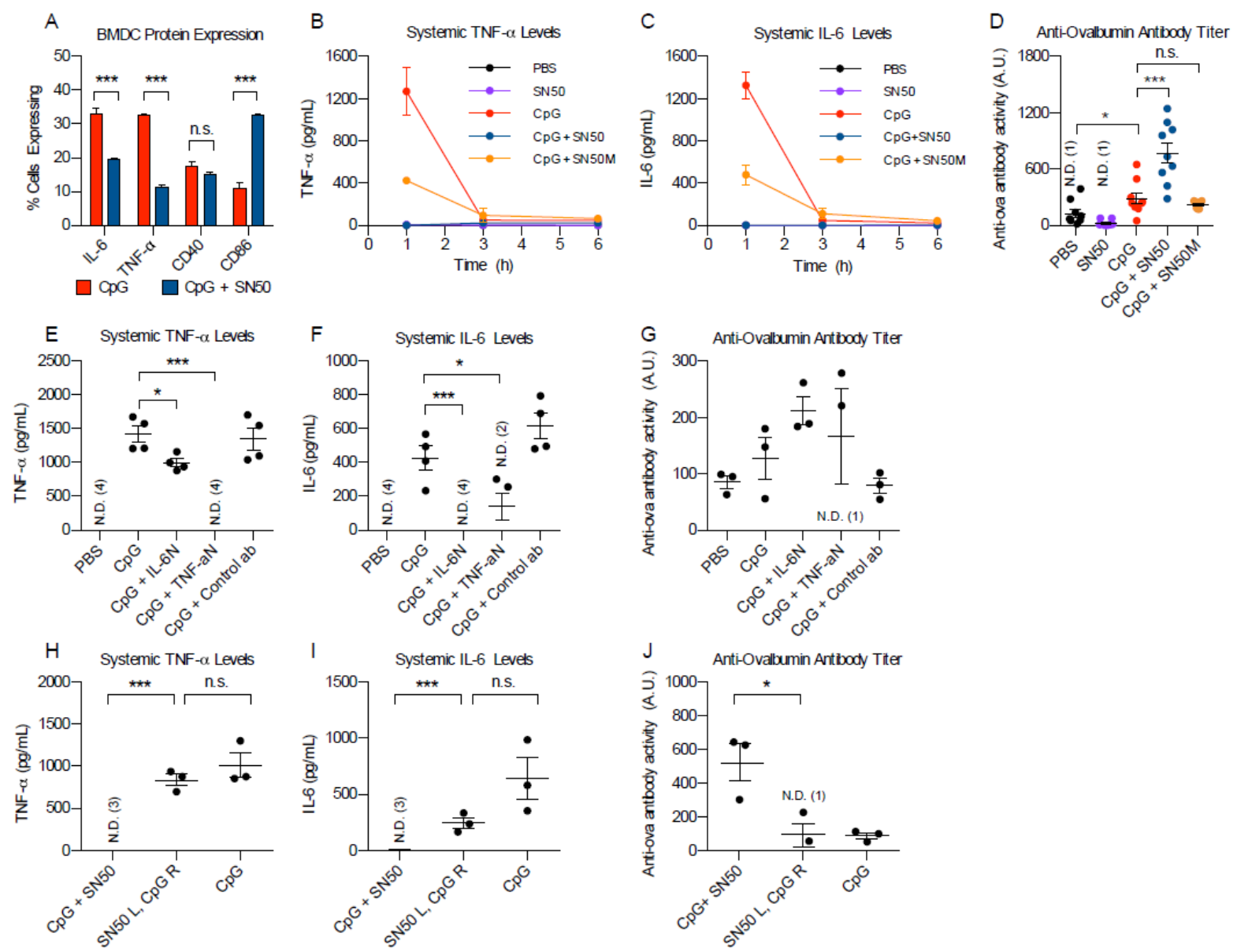

Figure 1. In vivo vaccination with model antigen ovalbumin and immune adjuvant SN50. (A) Intracellular cytokine staining of BMDCs treated with $\mathrm{CpG}$ (red bars) or CpG + SN50 (blue bars). (B) Systemic cytokine levels of TNF-a measured at $1 \mathrm{~h}, 3 \mathrm{~h}, 6 \mathrm{~h}$ post-injection with: PBS (black line), SN50 alone (purple line), CpG (red line), $\mathrm{CpG}+\mathrm{SN} 50$ (blue line), $\mathrm{CpG}+\mathrm{SN} 50 \mathrm{M}$ (yellow line), $\mathrm{n}=4$ for each time point. (C) Systemic cytokine levels of IL6. (D) Anti-ovalbumin antibody titer, day $28, \mathrm{n}=8$. (E) Systemic TNF-a levels $1 \mathrm{~h}$ post-vaccination with $\mathrm{CpG}, \mathrm{CpG}$ + IL-6N, CpG + TNF-aN or CpG + Control ab, $\mathrm{n}=4$. (F) Systemic IL-6 levels $1 \mathrm{~h}$ post-vaccination with CpG, $\mathrm{CpG}$ + IL-6N, CpG + TNF-aN or CpG + Control ab. (G) Anti-ovalbumin antibody titer, day 28. (H) Systemic TNF-a levels in mice vaccinated with mixed CpG and SN50 (CpG + SN50), SN50 in left limb and CpG + OVA in right limb (SN50L, CpG R, or CpG alone, n =3. (I) Systemic IL-6 levels. (J) Anti-OVA antibody titer, day 28.

\section{Examination of CpG-induced inflammation and resulting immune response}

We sought to verify that SN50 could enable antigen presenting cells to upregulate cell surface receptors, while limiting pro-inflammatory cytokine production. We incubated murine bone marrow- derived dendritic cells (BMDCs) with SN50 and $\mathrm{CpG}$ or $\mathrm{CpG}$ alone for 6h and analyzed how the potentiator altered cytokine production and cell surface receptor expression (Fig. 1a). Intracellular cytokine staining revealed that cells treated with SN50 demonstrated a $21 \%$ 
decrease in cells expressing TNF-a and a 13\% decrease in cells expressing IL-6. Meanwhile, CD86 was upregulated by $22 \%$ and CD40 was only down regulated by $2.5 \%$. Because the p65-p50 dimer is the most abundant dimer found in resting cells and involved in inflammatory cytokine production, we conjecture that by inhibiting this dimer, we enable the transcription and translation of cell surface receptors while limiting inflammatory cytokines. This is consistent with previous knockout experiments (36). The result is lower inflammatory responses while priming effective adaptive immune communication.

After observing that SN50 can limit inflammation without decreasing cell surface receptor expression in vitro, we next wanted to examine the effect in vivo. To determine if inhibition of NF-kB could decrease the systemic levels of pro-inflammatory cytokines associated with $\mathrm{CpG}$ vaccination, we vaccinated mice intramuscularly (i.m.) with $100 \mu \mathrm{g}$ ovalbumin (OVA) and: PBS, SN50 $(500 \mu \mathrm{g}), \mathrm{CpG}(50 \mu \mathrm{g}), \mathrm{SN} 50+\mathrm{CpG}$, or SN50M $(500 \mu \mathrm{g})+\mathrm{CpG}$. SN50M is a physical control for SN50 as it is a much weaker inhibitor. We chose to measure systemic levels of proinflammatory cytokines TNF-a and IL-6 because high levels are unsafe and lead to side effects $(17,18,38)$. We measured these pro-inflammatory cytokines at $1 \mathrm{~h}, 3 \mathrm{~h}, 6 \mathrm{~h}, 24 \mathrm{~h}$ and $48 \mathrm{~h}$ postinjection in all groups to determine the timepoint where cytokines peak in response to $\mathrm{CpG}$ vaccination (Fig. 1b, 1c, S2a, S2b). Mice vaccinated with OVA and PBS or SN50 alone elicited no systemic cytokine response. $\mathrm{CpG}$ demonstrated the highest response of both TNF-a (1325 pg/mL) and IL-6 (1269 pg/mL) at the $1 \mathrm{~h}$ timepoint. The CpG + SN50 group showed complete elimination of cytokines for both cytokines. The $\mathrm{CpG}+\mathrm{SN50M}$ group showed a decrease in cytokine levels, although not as large as observed with $\mathrm{CpG}+\mathrm{SN} 50$. We confirmed that this decrease in inflammatory cytokines is due to the high local inhibition of injected SN50M and not physical aggregation (Fig. S3). To determine how SN50 would affect the humoral response, we analyzed serum antibody levels on day 28 (Fig. 1d). The CpG group demonstrated a 2.4-fold increase in anti-OVA antibodies compared to PBS alone. Mice vaccinated with $\mathrm{CpG}+\mathrm{SN50}$ demonstrated a 5.9-fold increase over the PBS group and 2.7-fold increase over the CpG group. These data confirmed our hypothesis that high levels of systemic TNF-a and IL-6 can be decoupled from the humoral, adaptive immune response. We were surprised to find that addition of SN50 boosted the downstream adaptive response, leading to immune potentiation. Due to this increase in adaptive response and improved safety profile after vaccination we consider SN50 to be an immune potentiator. 


\section{Determining mechanism of action}

To more directly examine how early systemic expression of TNF-a and IL-6 impact the immediate inflammatory response and downstream adaptive response, we vaccinated mice with CpG and either TNF-a neutralizing antibody (TNF-aN) or IL-6 neutralizing antibody (IL-6N) and measured the systemic cytokines (Fig. 1e, 1f). The CpG + IL-6N group demonstrated a 1.4 -fold decrease in TNF-a expression and a complete reduction of systemic IL-6 expression. The CpG + TNF-aN group demonstrated complete elimination of systemic TNF-a and a 3-fold reduction of IL-6 expression. This result was confirmed by a control isotype antibody to rule out any nonspecific interactions. Although both IL-6N and TNF-aN groups demonstrated higher average antibody titer, these differences were not statistically significant (Fig. 1g). This indicates that reducing inflammation from $\mathrm{CpG}$ with the initial vaccination is not detrimental to antibody titer.

Upon observing this in vivo modulation, we sought to determine whether SN50 acts locally or systemically. To examine this mechanism, we injected SN50 i.m. in the left hind limb and immediately injected $\mathrm{CpG}+\mathrm{OVA}(\mathrm{SN} 50 \mathrm{~L}+\mathrm{CpG} \mathrm{R})$ in the right hind limb. There was no significant difference in systemic cytokine levels tested between CpG and the SN50 L + CpG R group (Figure 1h, 1i), whereas SN50 + CpG injected simultaneously demonstrated reduction of TNF-a and IL-6. On day 28, we analyzed antibody titer, reveling a 5.5 fold difference between the SN50 + CpG and the SN50 L +CpG R group (Fig. 1j). This demonstrates the importance of coadministration of the components and therefore indicates that SN50 is acting locally to both increase safety and protection.

From these experiments, we conclude that SN50 acts locally at the injection site to inhibit immediate cytokine production, containing inflammation before it is distributed systemically. Based on our in vitro data, we believe CpG + SN50 enables TNF-a and IL-6 production locally at reduced levels. Our in vitro data suggests that immune cells exposed to $\mathrm{CpG}+\mathrm{SN} 50$ express higher levels of cell surface receptors important for antigen presentation and effective $\mathrm{T}$ cell activation. While our experiments confirm that SN50 reduces systemic inflammation and increases antibody titer in vivo, more in depth exploration needs to be completed to fully understand our hypothesized mechanism. 
A

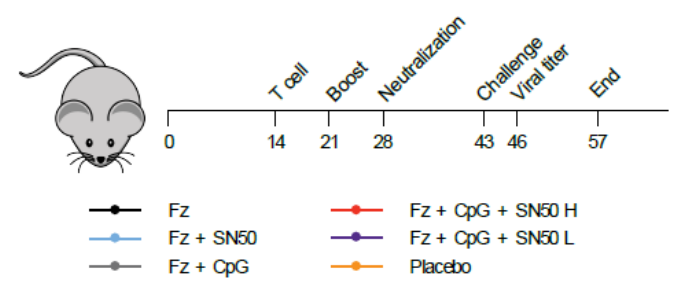

D

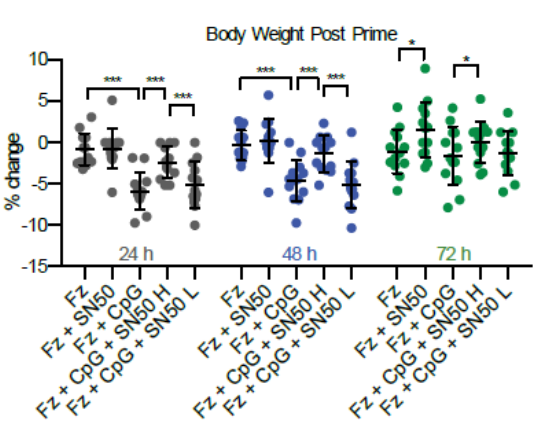

$\mathrm{H}$

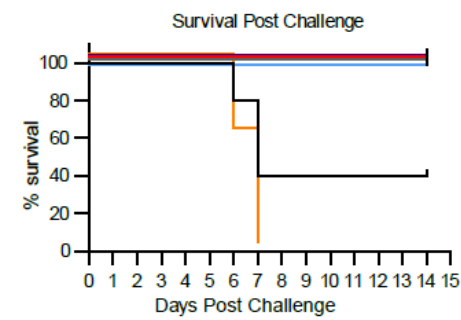

$\mathrm{K}$

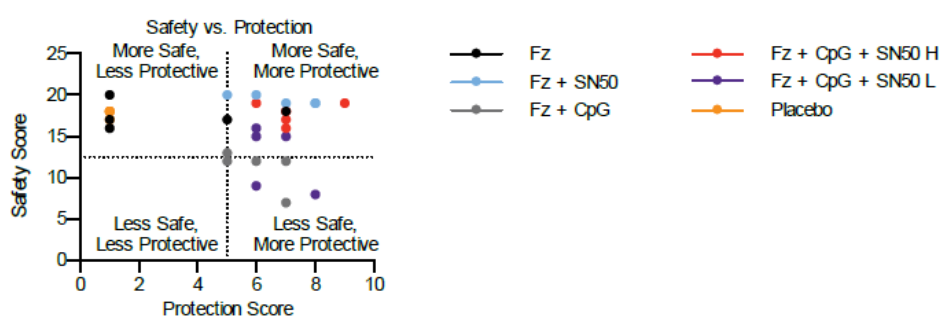

B

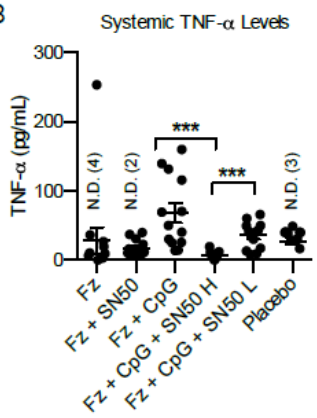

$E$

CD4+ IL-4+ Splenocytes

$\mathrm{F}$
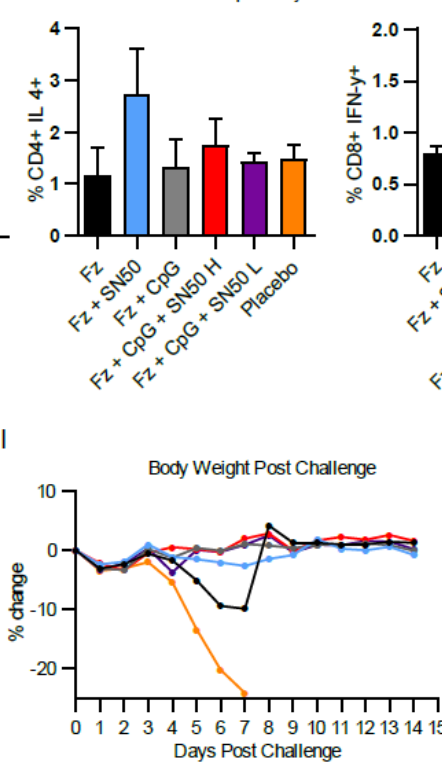

$\mathrm{J}$

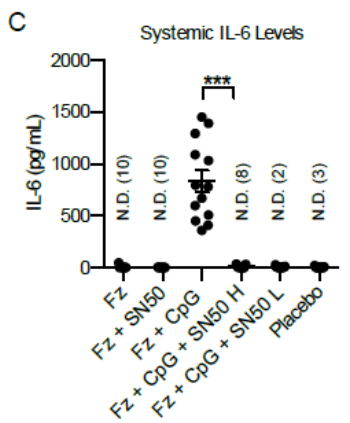

G

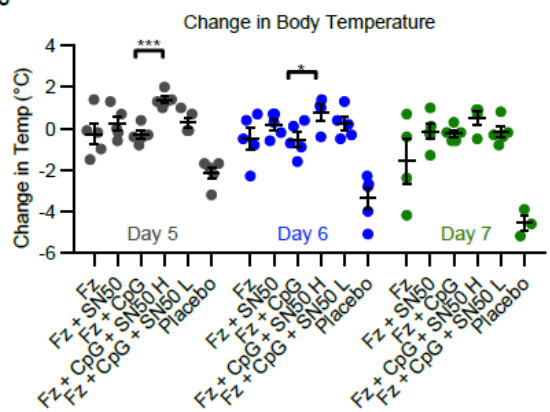

Figure 2. Influenza Challenge Model (A) Schematic of influenza challenge study. (B) Systemic TNF-a levels $1 \mathrm{~h}$ post-vaccination with $\mathrm{Fz}, \mathrm{Fz}+\mathrm{SN} 50, \mathrm{Fz}+\mathrm{CpG}, \mathrm{Fz}+\mathrm{CpG}+\mathrm{SN} 50 \mathrm{H}, \mathrm{Fz}+\mathrm{CpG}+\mathrm{SN} 50 \mathrm{~L}$, Placebo. n = 13 (C) Systemic IL-6 levels $1 \mathrm{~h}$ post-vaccination. $\mathrm{n}=13$ (D) Percent change in body weight $24 \mathrm{~h}$ (grey), 48h (blue) and $72 \mathrm{~h}$ (green) post-vaccination, $\mathrm{n}=13$. (E) Day $28 \mathrm{IgG}$ antibody titer, $\mathrm{n}=8$. (F) Survival 1-14 days post challenge, $\mathrm{n}=5$. Groups: Fz (black), Fz + SN50 (blue), Fz + CpG (grey), Fz + CpG + SN50 H (red), Fz + CpG + SN50 L (purple), Placebo (orange). $n=5(\mathrm{G})$ Percent change in body weight 1-14 days, $n=5$. $(\mathrm{H})$ Body temperature 1-14 days post challenge, $n=5$. (I) Safety vs Protection score. 


\section{Immune potentiation in in vivo influenza challenge model}

We next wanted to focus on how SN50 might transition to a vaccine with challenge. We selected influenza vaccine as a proof-of-concept vaccination both due to its universality and the relative ease of running animal challenges with multiple parameters. We sought to determine if SN50 would reduce side effects associated with strong adjuvanticity and to see what effect this alteration on systemic cytokines would have on protection. We vaccinated mice i.m. with Fluzone ${ }^{\circledR}$ quadrivalent vaccine (Fz) for the 2017/2018 influenza season, with or without CpG (50 $\mu \mathrm{g})$ as an immune adjuvant and $500 \mu \mathrm{g} \mathrm{SN} 50$ (SN50 H) or $50 \mu \mathrm{g}$ SN50 (SN50 L)) as an immune potentiator. The Fz + SN50 group demonstrated lower levels of TNF-a than Fz alone (Fig. 2b, 2c). Across all groups, the addition of SN50 reduced levels of TNF-a and IL-6 to levels consistent with the placebo group. To examine whether SN50 can mitigate side effects from vaccination, we analyzed the percent change in body weight 24, 48 and 72 h post-vaccination (Fig. 2d, S4). Weight loss is the easiest and most objective measure of side effects in mice. Mice vaccinated with Fz and $\mathrm{Fz}+\mathrm{SN} 50$ lost an average of $0.85 \%$ and $0.75 \%$, respectively by the $24 \mathrm{~h}$ timepoint. The $\mathrm{Fz}+\mathrm{CpG}$ group lost an average of 5.9\%. Adding SN50 H decreased the amount of weight loss to $2.4 \%$ and SN50 L to $5.1 \%$. At $72 \mathrm{~h}$ the Fz group were $-1.1 \%$ of the starting weight whereas mice vaccinated with $\mathrm{Fz}+\mathrm{SN} 50$ gained $+1.5 \%$. The $\mathrm{Fz}+\mathrm{CpG}$ group lost $-1.6 \%$ of the starting weight and adding SN50 H lead to a reduction in weight loss (0\% change) and adding SN50 L lead to $-1.3 \%$ change. Overall, mice with SN50 lost less weight than mice without SN50, demonstrating that SN50 lowered side effects associated with vaccination.

We next wanted to see if the addition of SN50 would change the T cell responses or antibody titer. On day 14, we analyzed splenocytes for antigen specific CD4+ and CD8+ T cells. We observed no statistically significant differences between samples with and without SN50 (Fig. 2e, 2f). On day 28, we analyzed the serum for antibody levels in the blood (Fig. 2g and S5-7). There was no significant difference in IgG titer between $\mathrm{Fz}$ and Fz + SN50. There was a significant difference between $\mathrm{Fz}$ samples and $\mathrm{Fz}+\mathrm{CpG}$ of 2.9 fold. There was no significant difference between groups vaccinated with $\mathrm{CpG}$, implying that the addition of SN50 reduces inflammation and side effects from vaccination, while maintaining the antibody titer.

We next sought to determine if SN50 would increase the protection of Fluzone. Mice were lethally challenged intranasally with $10^{5} \mathrm{PFU}$ A/Michigan/45/2015. On day 3 post-challenge we 
analyzed the lungs of three mice for viral titer (Fig. S8). Survival was analyzed for 14 days postchallenge (Fig. 2h). By day 7, all placebo mice and $60 \%$ of the Fz mice had reached the humane endpoint and were euthanized. All other mice survived. The Fz + SN50 group was significantly more protected than the $\mathrm{Fz}$ alone group. The addition of $\mathrm{SN} 50$ to $\mathrm{Fz}+\mathrm{CpG}$ confers equal protection, while improving side effects from the initial vaccination. Surprisingly, simply adding $\mathrm{SN} 50$ to Fz conferred enhanced protection equal to $\mathrm{Fz}+\mathrm{CpG}$ group.

Mice were analyzed for change in body weight and body temperature for 14 days postchallenge (Fig. 2i, 2j, Fig. S9). The peak average weight loss between Fz (-9.9\%) and Fz + SN50 $(-2.67 \%)$ was statistically significant. Greater weight loss is associated with a more intense infection, these data demonstrate that adding SN50 to Fz improves the response to infection. Addition of SN50 to $\mathrm{Fz}+\mathrm{CpG}$ demonstrates no significant change in weight loss indicating that the SN50 can reduce systemic cytokines and side effects from vaccination with no detrimental effects to the protective response.

As an additional parameter of disease pathology, we examined body temperature postchallenge. Unlike in humans, mice demonstrate a reduction in body temperature upon infection (39). The placebo has the largest peak drop in temperature $\left(-4.57^{\circ} \mathrm{C}\right)$, followed by the Fz group ( $-1.58{ }^{\circ} \mathrm{C}$ ) (Fig. 2j). Adding SN50 to $\mathrm{Fz}$ or $\mathrm{Fz}+\mathrm{CpG}$ mitigated the decrease in temperature across all groups.

Safety and protection of new vaccine adjuvants are typically considered two interdependent variables with an inverse relationship, where adequate protection is acquired by limiting safety or vice versa. As this potentiator makes the vaccine both safer and more protective, we sought a single way to analyze how SN50 was changing the safety and protection profile. As these variables are considered inversely related, there are few precedents for correlation. However, a common scoring system used widely across fields is a quartile-based scoring system (40-43). Following precedent for scoring systems, we developed a safety vs protection plot (Fig. 2k). This plot is meant only to serve as a visual representation of all data collected within this study. All groups that included SN50 in the vaccination increased both the safety and the protection of the vaccine. When all data is taken together, we conclude that SN50 acts as an immune potentiator by both increasing the safety profile and improving the protective outcome of the vaccination.

Next, we wanted to examine if this type of immune potentiator could improve safety and maintain the adaptive response across a broader range of diseases and antigens. We chose to 
vaccinate against dengue and HIV because they represent additional, important diseases with active vaccine research. In each case, challenges with current methods have been identified and we wanted to see if SN50 could help address those challenges, as well as maintain the current function of vaccination strategies. For dengue, the main challenge is producing antibodies that neutralize the virus, inhibiting cellular uptake. For HIV, a key challenge is in generating $\operatorname{IgA}$ antibodies at the mucosal interface as well as eliciting broadly neutralizing antibodies targeted to select epitopes. To explore how adding an immune potentiator affects each of these responses, we analyzed each antigen set in greater detail. 

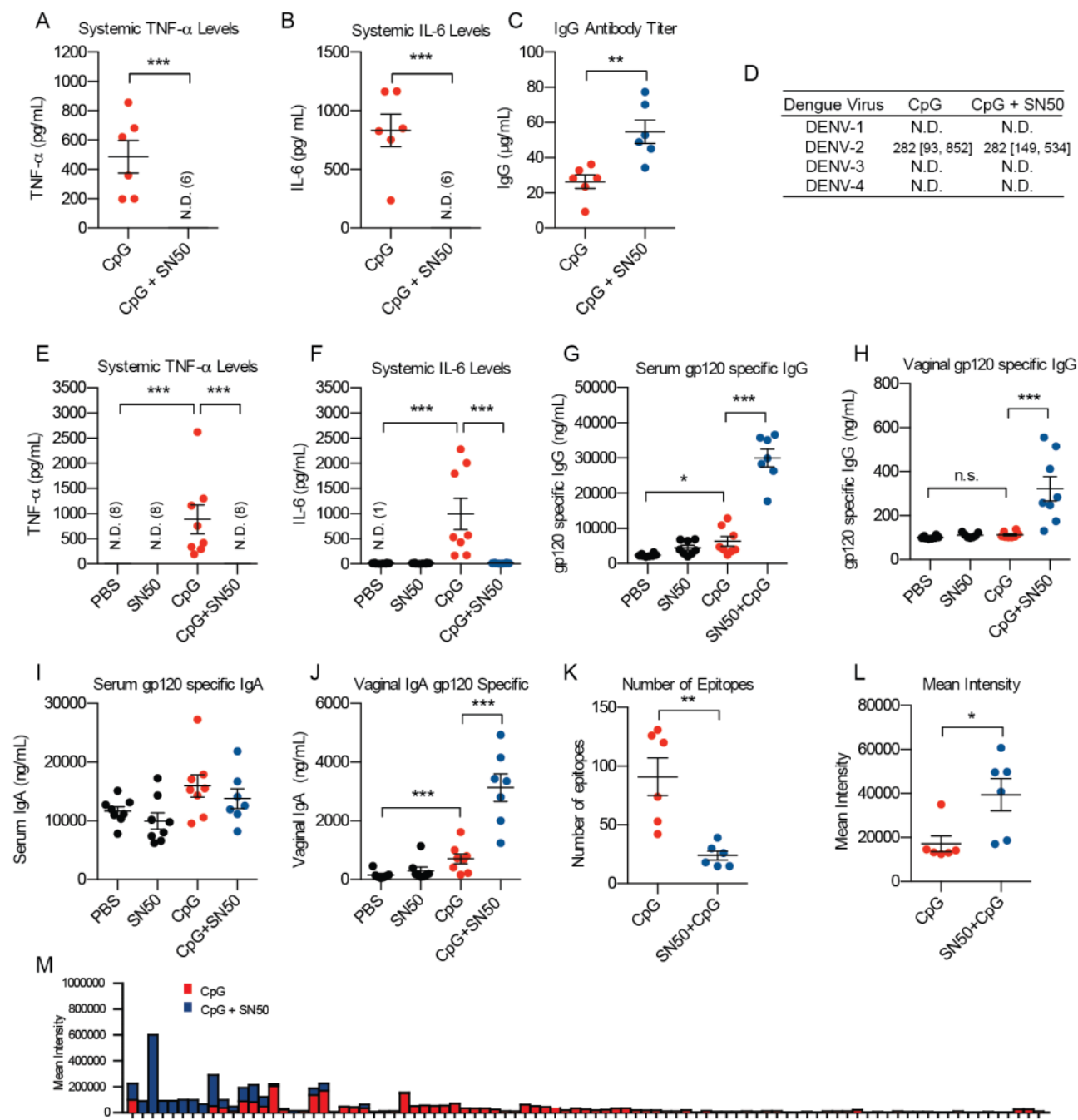

Figure 3. In vivo vaccination against dengue and HIV (A) Systemic TNF-a levels $1 \mathrm{~h}$ post-vaccination with DENV$2 \mathrm{C}$ antigen and $\mathrm{CpG}$ or $\mathrm{CpG}+\mathrm{SN} 50, \mathrm{n}=6$. (B) Systemic IL-6 levels $1 \mathrm{~h}$ post-vaccination with DENV-2C antigen and $\mathrm{CpG}$ or $\mathrm{CpG}+\mathrm{SN50}$. (C) IgG antibody titer day 28 post vaccination with DENV-2C antigen. (D) Dengue virus neutralization. Geometric mean [95\% confidence interval]. (E) Systemic TNF-a levels measured at $1 \mathrm{~h}$ post-injection with gp120 and: PBS, CpG, SN50, SN50 + CpG, n = 8 (B) Systemic IL-6 levels measured at $1 \mathrm{~h}$ post-injection with gp120 vaccinations (C) Serum anti-gp120 IgG antibody titer, day 28 after vaccination with gp120. (D) Vaginal antigp120 IgG antibody titer, day 28. (E) Serum anti-gp120 IgA antibody titer, day 28. (F) Vaginal anti-gp120 IgA antibody titer, day 28. (G) Number of g120 epitopes recognized by mice vaccinated with CpG or SN50 + CpG. (H) Mean intensity of recognized epitopes. (I) Mean intensity of each recognized epitope by $\mathrm{CpG}$ (red bars) or $\mathrm{CpG}+$ SN50 (blue bars). 


\section{Examination of immune potentiator on Dengue neutralization}

To explore dengue further, we vaccinated mice with the capsid protein of dengue serotype 2 (DENV-2C) and: CpG $(50 \mu \mathrm{g}), \mathrm{CpG}+500 \mu \mathrm{g}$ SN50. SN50 completely eliminated expression of systemic cytokines (Fig. 3a, 3b). On day 28 we analyzed the difference in antibody titer (Fig. 3c). Antibody titer in $\mathrm{CpG}+\mathrm{SN} 50$ mice were almost two-fold higher than the $\mathrm{CpG}$ group.

To determine if SN50 alters the neutralization potential, we analyzed the neutralizing titer for four strains of dengue (Fig. 3d). We tested four serum samples against one strain representative of each dengue serotype. The differences in neutralization potential were not significantly different between the two groups implying that, similar to our flu results, SN50 improves the safety while maintaining the protective responses of vaccination.

\section{Analysis of influence of immune potentiator on HIV vaccination}

To further test the efficacy of vaccines with SN50 and to attain a broader picture of the induced humoral immune response, we vaccinated mice with gp120, a viral coat protein from HIV necessary for infection and a target of many HIV vaccines, using $\mathrm{CpG}$ as the immune adjuvant. Mice vaccinated with CpG demonstrated high levels of both TNF-a and IL-6, whereas all other groups including mice vaccinated with $\mathrm{CpG}+\mathrm{SN} 50$ demonstrated non-detectable levels of systemic cytokines at the $1 \mathrm{~h}$ time point (Fig. 3e, 3f). On day 28 , we analyzed antibody titers against gp120. The $\mathrm{CpG}+\mathrm{SN} 50$ group induced a 4.7 fold higher anti-gp120 IgG antibody titer than the CpG group in the serum (Fig. 3g, 3h). This demonstrates that the addition of SN50 increases IgG antibody titer across multiple antigens and suggests that it may serve as a general immune potentiator. Because mucous membranes are particularly susceptible to HIV infection, we also measured the anti-gp120 IgG and IgA antibody titers in vaginal secretions (Fig. 3i, 3j). The CpG + SN50 group demonstrated a 4.4 fold increase in anti-gp120 IgA antibodies than mice vaccinated with CpG alone. These results suggest that SN50 with gp120 may help induce class-switching to IgA antibody isotype, while also enabling localization to the mucous membranes.

We next chose to determine if there were any alterations in the gp120 epitopes recognized by the resulting antibodies, using an overlapping peptide microarray. Interestingly, the number of epitopes recognized by $\mathrm{CpG}$ alone was higher than antibodies collected from $\mathrm{CpG}+\mathrm{SN} 50$ mice; however, the fluorescent mean intensity of recognized epitopes is higher in the $\mathrm{CpG}+\mathrm{SN} 50$ mice (Fig. 3k, 3l) - implying a higher concentration of antibodies against those epitopes. Upon closer inspection of particular epitopes recognized, we saw that adding the immune potentiator to the 
formulation shifts the epitope recognition, as different epitopes are recognized in the $\mathrm{CpG}$ alone and $\mathrm{CpG}+\mathrm{SN50}$, often exclusively in one condition or the other (Fig. 3m). This may prove valuable with diseases where the current recognized epitopes are not effective enough to provide protection. The most highly recognized epitope in the $\mathrm{CpG}+\mathrm{SN50}$ group corresponds to the epitope recognized by the recently isolated $35 \mathrm{O} 22$ monoclonal antibody (46). Antibodies isolated from mice vaccinated with $\mathrm{CpG}+\mathrm{SN} 50$ also recognize the CD4 binding site recognized by several potent, broadly neutralizing antibodies (VRC 01, VRC03, b12). From these data we demonstrate that the addition of SN50 shifts the epitope selectivity in the case of gp120. Based on the epitopes recognized by the serum samples, we hypothesize that these antibodies may be more broadly neutralizing. 

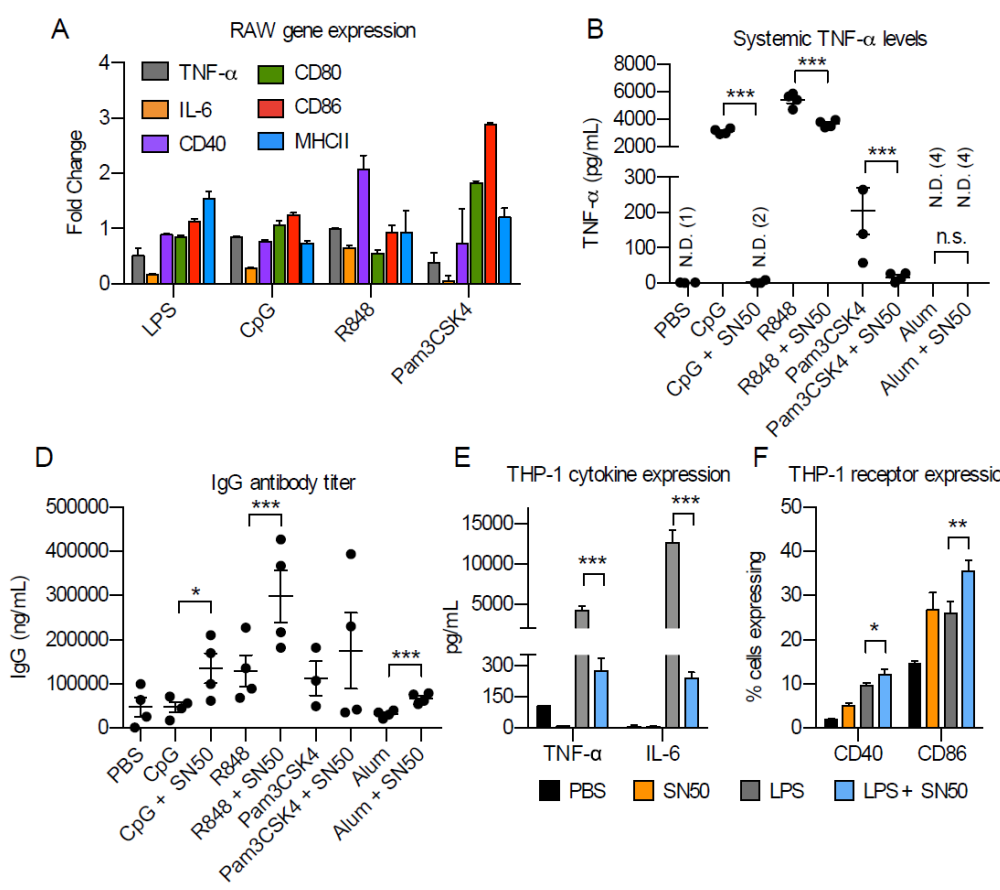

E THP-1 cytokine expression
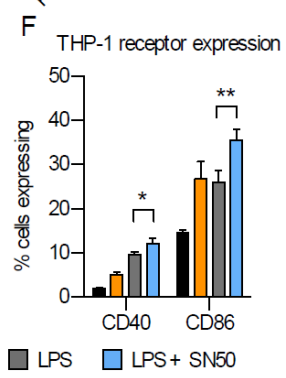
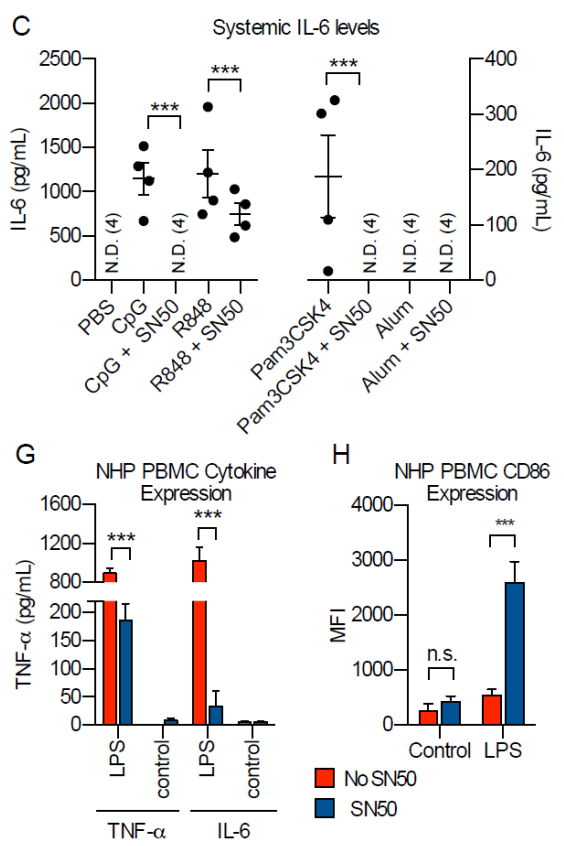

H NHP PBMC CD86

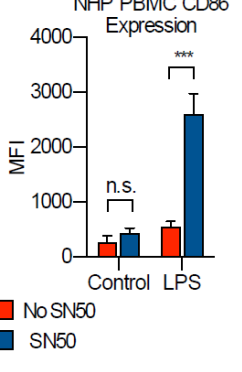

Figure 4. In vivo vaccinations across a broad range of adjuvants. (A) qPCR gene expression analysis of RAW macrophages stimulated with SN50 and TLR agonists compared to cells stimulated with TLR agonist alone. Proinflammatory cytokines TNF-a (grey bars) and IL-6 (orange bars) and cell surface receptors CD40 (purple bars), CD80 (green bars), CD86 (red bars) and MHCII (blue bars). (B) Systemic TNF-a cytokine levels of TNF-a measured at 1h post-injection with gp120 and: PBS, CpG, CpG + SN50, Pam3CSK4, Pam3CSK4 + SN50, R848, R848 + SN50, Alum, Alum + SN50, $\mathrm{n}=4$. (C) Systemic IL-6 cytokine levels measured at $1 \mathrm{~h}$ post-injection. (D) Serum IgG antibody titers, day 28. (E) Human THP-1 cell pro-inflammatory cytokines TNF-a and IL-6 in cell supernatant after treatment with PBS (black bars), SN50 (orange bars), LPS (grey bars), or LPS + SN50 (blue bars). (F) Cell surface receptor expression on human THP-1 cell after treatment with PBS (black bars), SN50 (orange bars), LPS (grey bars), or LPS + SN50 (blue bars). (G) Cytokine expression analysis of TNF-a and IL-6 in cell supernatant of NHP PBMCs 6h. No SN50 (red bars), SN50 (blue bars). LPS $1 \mu \mathrm{g} / \mathrm{mL}$ (H) CD86 expression of NHP PBMCs 18h. No SN50 (red bars), SN50 (blue bars).

\section{Improvement of adjuvant responses across a variety of TLRs and Species}

To examine the effects of the SN50 across a broad range of TLR agonists, we performed qPCR on RAW macrophages treated with SN50 followed by stimulation with agonists of different TLRs. We stimulated cells with SN50 and LPS (10 ng/mL), CpG $(5 \mu \mathrm{g} / \mathrm{mL}), \mathrm{R} 848(1 \mu \mathrm{g} / \mathrm{mL})$ and Pam3CSK4 (100 ng/mL) and compared transcript levels to cells treated with TLR agonist alone

(Fig. 4a). We chose these TLR agonists because they represent a subset of the compounds with promising potential for commercial use if the inflammatory side effects can be controlled. In RAW macrophages, we observed downregulation of TNF-a and IL-6 pro-inflammatory cytokine transcript levels. Across all agonists, the cell surface receptors CD86 and MHCII transcript levels were upregulated, compared to agonist alone, implying that cellular communication of the APC to 
the T cell may not be attenuated by the addition of SN50 and subsequent reduction in cytokine production.

To examine how this would translate in vivo, we vaccinated mice with $\mathrm{CpG}(50 \mu \mathrm{g})$, Pam3CSK4 $(20 \mu \mathrm{g})$ and R848 (50 $\mu \mathrm{g})$ using gp120 as the antigen. We chose to run these adjuvants alongside the most widely employed adjuvant, alum $(250 \mu \mathrm{g})$.

With CpG, we observed complete elimination of systemic TNF-a and IL-6 proinflammatory cytokines (Fig. 4b, 4c). With R848 and Pam3CSK4 we saw a significant decrease in systemic cytokines. We hypothesize that SN50 is less effective at decreasing cytokines with R848 due to the low molecular weight of the molecule, enabling more rapid systemic distribution. Alum alone did not evoke a systemic cytokine response and the addition of SN50 did not alter the cytokine profile. The addition of SN50 increased the antibody titer for all adjuvants, including alum, demonstrating the broad potential use of this system to a large number of immune adjuvants (Fig. 4d).

To understand how this may translate to human vaccinations, we treated THP-1 monocytes with $1 \mu \mathrm{g} / \mathrm{mL}$ LPS with or without SN50. Cells treated with SN50 and LPS expressed dramatically lower levels of TNF-a and IL-6 (Fig. 4e). We also observed increased levels of CD40 and CD86 (Fig. 4f). Additionally, we examined the effects of SN50 on non-human primate rhesus macaque (NHP) primary peripheral blood mononuclear cells (PBMCs). We stimulated NHP PBMCs with SN50 and LPS or LPS alone for 6h and analyzed the cell supernatant for pro-inflammatory cytokines. Cells stimulated with LPS demonstrated high levels of TNF-a and IL-6 in the cell supernatant, cells with SN50 demonstrated significant reduction in cytokine levels (Fig. 4g). We observed that CD86 expression was upregulated 2-fold in cells stimulated with SN50 and LPS compared to cells stimulated with LPS alone (Fig. 4h). This implies that SN50 may work similarly in NHP and humans as it does in mice.

\section{CONCLUSION}

Using a broad range of TLR agonists, we show both in vitro and in vivo that a cell permeable inhibitor of the p50 subunit of NF-kB, potentiates the immune response - reducing inflammation while increasing antibody responses. Co-administration of $\mathrm{CpG}$ with the immune potentiator 30 results in significantly reduced levels of proinflammatory cytokines, often at undetectable levels. At the same time, this reduction in inflammation results in a 3 -fold increase in the IgG titer of 
antibodies for the model antigen OVA. We examined how potentiation would enhance the capabilities of the adjuvants to improve the immune response. In our influenza model we directly examined side effects in response to the current commercial flu vaccine and $\mathrm{Fz}+\mathrm{CpG}$ and determine that adding SN50 reduces side effects and systemic pro-inflammatory cytokine levels. We also demonstrate that the safety profile can be enhanced without negatively effecting the protective response. After vaccinated mice were challenged with influenza A, mice with SN50 added to the vaccine, lead to increased survival, less weight loss and less change in body temperature. To study the effects of potentiation on TLR agonists as vaccine adjuvants, we selected three diseases - influenza, dengue and HIV - all of which have had different challenges in vaccine development. In dengue vaccination, the goal is to increase antibody neutralization potential while maintaining a safe profile. We demonstrate that there are no detrimental effects to dengue neutralization of antibodies with SN50, enabling us to mitigate side effects but maintain the protective response. In HIV, we vaccinated with HIV envelope protein gp120, CpG and SN50, increased both IgG and IgA titers. This method appears quite general as it works with many TLR agonists and antigens. SN50 is one of hundreds of similar NF-kB inhibitors. When used in combination with the appropriate TLR agonist, many may prove useful for eliciting specific and potentially tunable responses for distinct vaccines or immunotherapies. This methodology may find use in reducing the systemic side effects associated with inflammation seen in many adjuvanted vaccines (19). This method has the potential to enable a variety of PRR agonists to be used safely in vaccines, increasing the diversity of adaptive immune profiles and widening the scope of disease prevention and treatment.

In conclusion, we have demonstrated that using a specific NF-kB inhibitor in combination with common immune adjuvants can decrease pro-inflammatory cytokine production while boosting cell-surface receptor expression for effective antigen presentation and $\mathrm{T}$ cell activation in mouse, human and NHP primary cells. The use of this inhibitor in vivo completely reduced systemic TNF-a and IL-6 to baseline levels while increasing the downstream adaptive humoral response from the vaccination. These phenomena were observed across a broad range of antigens for a variety of pathogens demonstrating that this may prove a general strategy for improving vaccination response while conforming to strict safety standards. There are hundreds of documented immune adjuvants that provide adequate protection against diseases but induce unsafe levels of inflammation to be approved for clinical use $(7,45)$. Additionally there are hundreds of 
NF-kB inhibitors, some already with FDA approval, that could be multiplexed with different TLR agonists to provide a broad range of responses (44). We anticipate this framework will enable a variety of TLR agonists to be used safely in human vaccines, increasing the diversity of adaptive immune profiles and widening the scope of disease prevention and treatment.

\section{References and Notes:}

1. Petrovsky, N. \& Aguilar, J. C. Vaccine adjuvants: Current state and future trends. Immunol. Cell Biol. 82, 488-496 (2004).

2. Coffman, R. L., Sher, A. \& Seder, R. A. Vaccine Adjuvants: Putting Innate Immunity to Work. Immunity 33, 492-503 (2010).

3. Pashine, A., Valiante, N. M. \& Ulmer, J. B. Targeting the innate immune response with improved vaccine adjuvants. Nat. Med. 11, S63-S68 (2005).

4. Hoebe, K., Janssen, E. \& Beutler, B. The interface between innate and adaptive immunity. Nature Immunology (2004). doi:10.1038/ni1004-971 CD103+ CD11b+ Brain Dendritic Cell Populations Within the Olfactory Bulb. Proc. Natl. Acad. Sci. 109, 6175-6180 (2012).

6. Banchereau, J. \& Steinman, R. M. Dendritic cells and the control of immunity. Nature 392, 245-252 (1998).

7. van Duin, D., Medzhitov, R. \& Shaw, A. C. Triggering TLR signaling in vaccination. Trends Immunol. 27, 49-55 (2006).

8. Dale, C. J. et al. Evaluation in macaques of HIV-1 DNA vaccines containing primate $\mathrm{CpG}$ motifs and fowlpoxvirus vaccines co-expressing IFN $\gamma$ or IL-12. Vaccine 23, 188-197 (2004).

9. Weeratna, R., Comanita, L. \& Davis, H. L. CPG ODN allows lower dose of antigen against hepatitis B surface antigen in BALB/c mice. Immunol. Cell Biol. 81, 59-62 (2003).

10. Klinman, D. M., Xie, H. \& Ivins, B. E. CpG oligonucleotides improve the protective immune response induced by the licensed anthrax vaccine. Ann. N. Y. Acad. Sci. 1082, 137-150 (2006). lymphocyte epitope peptide vaccination, CpG-oligodeoxynucleotide adjuvant, and CTLA-4 blockade. - Semantic Scholar. Cancer Res. 63(12), 3281-8 (2003).

12. Krieg, A. M. Toll-like receptor 9 (TLR9) agonists in the treatment of cancer. Oncogene 27, 161-167 (2008). 
13. Bode, C., Zhao, G., Steinhagen, F., Kinjo, T. \& Klinman, D. M. CpG DNA as a vaccine adjuvant. Expert Rev. Vaccines 10, 499-511 (2011).

14. Klinman, D. CpG DNA as a vaccine adjuvant: Expert Review of Vaccines Expert Review of Vaccines 2(2), 305-315 (2003).

15. Kanzler, H., Barrat, F. J., Hessel, E. M. \& Coffman, R. L. Therapeutic targeting of innate immunity with Toll-like receptor agonists and antagonists. Nat. Med. 13, 552-559 (2007).

16. Mbow, M. L., De Gregorio, E., Valiante, N. M. \& Rappuoli, R. New adjuvants for human vaccines. Curr. Opin. Immunol. 22, 411-416 (2010).

17. Christian, L. M., Porter, K., Karlsson, E. \& Schultz-Cherry, S. Proinflammatory cytokine responses correspond with subjective side effects after influenza virus vaccination. Vaccine 33, 3360-3366 (2015).

18. Simon, W. L., Salk, H. M., Ovsyannikova, I. G., Kennedy, R. B. \& Poland, G. A. Cytokine production associated with smallpox vaccine responses. Immunotherapy 6, 1097-1112 (2014).

19. Pasquale, A. D., Preiss, S., Silva, F. T. D. \& Garçon, N. Vaccine adjuvants: from 1920 to 2015 and beyond. Vaccines 3, 320-343 (2015).

20. Barton, G. M. \& Medzhitov, R. Toll-Like Receptor Signaling Pathways. Science 300, 1524-1525 (2003).

21. Lawrence, T. The Nuclear Factor NF- B Pathway in Inflammation. Cold Spring Harb. Perspect. Biol. 1, a001651-a001651 (2009).

22. Akira, S. \& Takeda, K. Toll-like receptor signalling. Nat. Rev. Immunol. 4, 499-511 (2004).

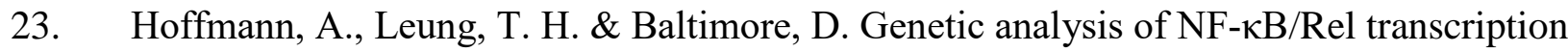
factors defines functional specificities. EMBO J. 22, 5530-5539 (2003). Rev. 210, 171-186 (2006).

25. Baeuerle, P. A. \& Henkel, T. Function and Activation of NF-kappaB in the Immune System. Annu. Rev. Immunol. 12, 141-179 (1994).

26. Dolcet, X., Llobet, D., Pallares, J. \& Matias-Guiu, X. NF-kB in development and progression of human cancer. Virchows Arch. 446, 475-482 (2005).

27. Wang, C.-Y., Mayo, M. W. \& Baldwin, A. S. TNF- and Cancer Therapy-Induced Apoptosis: Potentiation by Inhibition of NF-кB. Science 274, 784-787 (1996).

28. Xia, Y., Shen, S. \& Verma, I. M. NF-кB, an active player in human cancers. Cancer Immunol. Res. 2, 823-830 (2014). 
29. Erstad, D. J. \& Cusack, J. C. Targeting the NF-кB pathway in cancer therapy. Surg. Oncol. Clin. N. Am. 22, 705-746 (2013).

30. Bacher, S. \& Schmitz, M. L. The NF-kB Pathway as a Potential Target for Autoimmune Disease Therapy. Curr. Pharm. Des. 10(23), 2827-37 (2004).

31. Palanki, M. s s. Inhibitors of AP-1 and NF-kB Mediated Transcriptional Activation: Therapeutic Potential in Autoimmune Diseases and Structural Diversity. Curr. Med. Chem. 9(2) 219-227 (2002).

32. Letoha, T. et al. In Vitro and in Vivo Nuclear Factor- $\kappa B$ Inhibitory Effects of the CellPenetrating Penetratin Peptide. Mol. Pharmacol. 69, 2027-2036 (2006).

33. Liu, S. F., Ye, X. \& Malik, A. B. In vivo inhibition of nuclear factor-kappa B activation prevents inducible nitric oxide synthase expression and systemic hypotension in a rat model of septic shock. J. Immunol. 159, 3976-3983 (1997).

34. Eigler, A., Sinha, B., Hartmann, G. \& Endres, S. Taming TNF: strategies to restrain this proinflammatory cytokine. Immunol. Today 18, 487-492 (1997).

35. Tak, P. P. \& Firestein, G. S. NF- $\mathrm{BB}$ : a key role in inflammatory diseases. J. Clin. Invest. 107, 7-11 (2001).

36. Wang, J. et al. Distinct Roles of Different NF- $\kappa$ B Subunits in Regulating Inflammatory and T Cell Stimulatory Gene Expression in Dendritic Cells. J. Immunol. 178, 6777-6788 (2007).

37. Zienkiewicz, J.; Armitage, A.; Hawiger, J. Targeting Nuclear Import Shuttles, Importins/Karyopherins alpha by a Peptide Mimicking the NFkB1/p50 Nuclear Localization Sequence. J. Am. Heart Assoc. 2(5), e000386 (2013).

38. Netea, M. G., Kullberg, B. J., Meer, V. der \& M, J. W. Circulating Cytokines as Mediators of Fever. Clin. Infect. Dis. 31, S178-S184 (2000).

39. Bouvier, N. M. \& Lowen, A. C. Animal Models for Influenza Virus Pathogenesis and Transmission. Viruses 2, 1530-1563 (2010).

40. Daniele, G. et al. The inflammatory status score including IL-6, TNF- $\alpha$, osteopontin, fractalkine, MCP-1 and adiponectin underlies whole-body insulin resistance and hyperglycemia in type 2 diabetes mellitus. Acta Diabetol. 51, 123-131 (2014).

41. Segev, G., Kass, P. H., Francey, T. \& Cowgill, L. D. A Novel Clinical Scoring System for Outcome Prediction in Dogs with Acute Kidney Injury Managed by Hemodialysis. J. Vet. Intern. Med. 22, 301-308 (2008).

42. Page, K. M. Zhang, L.; Medizabal, A.; Wease, S.; Carter, S.; Shoulars, K.; Gentry, T.; Balber, A.; Kurtzberg, J. The Cord Blood Apgar: a novel scoring system to optimize selection of banked cord blood grafts for transplantation (CME). Transfusion (Paris) 52, 272-283 (2012). 
tolerance associated with lifestyle transition occurring in the rural population in India.

Diabetologia 47, 860-865 (2004).

44. Bhardwaj, N., Gnjatic, S. \& Sawhney, N. B. TLR AGONISTS: Are They Good Adjuvants? Cancer J. Sudbury Mass 16, 382-391 (2010).

46. Huang, J. et al. Broad and potent HIV-1 neutralization by a human antibody that binds the gp41-gp120 interface. Nature 515, 138-142 (2014).

Acknowledgments: The authors thank Prof. Amanda Burkhardt and Adrienne Gilkes for training and assistance with in vivo techniques. The authors thank Rie Nakajima for assistance imaging microarrays and Dan Piraner for help with image analysis. The authors thank Alfred Chon for assistance with HPLC optimization and Naorem Nihesh for training and assistance with peptide synthesis. The authors acknowledge Dr. Jin Qin at the University of Chicago for training and assistance with mass spectrometry.

Funding: We would like to acknowledge support by the NIH (1U01Al124286-01 and 1DP2A1112194-01, GM099594). Prof. Esser-Kahn thanks the Pew Scholars Program and the Cottrell Scholars Program for generous support. B.A.M. thanks NSF-GRFP (DGE-1321846). We would like to thank NSF instrumentation grant CHE-1048528. This work was supported, in part, by a grant from the Alfred P. Sloan foundation. Author contributions: B.A.M. and A.E.K conceived of and designed the project and experiments and wrote the manuscript. B.A.M., R.S., Y.E.B, D.B., K.B., B.G.M. performed experiments. B.A.M. and S.Y. synthesized materials. Competing interests: The authors report no competing interests. Data and materials availability: All data are available in the main text or the supplementary materials.

\section{Supplementary Materials:}

Materials and Methods

Figures S1-S9 